\title{
Correction to: Long-term study of a subdioecious Populus $\times$ canescens family reveals sex lability of females and reproduction behaviour of cosexual plants
}

\author{
Maurizio Sabatti ${ }^{1}$ (D) Muriel Gaudet ${ }^{2} \cdot$ Niels A. Müller $^{3} \cdot$ Birgit Kersten $^{3} \cdot$ Cosimo Gaudiano $^{1}$. \\ Giuseppe Scarascia Mugnozza ${ }^{1} \cdot$ Matthias Fladung $^{3} \cdot$ Isacco Beritognolo $^{2}$
}

Published online: 23 November 2019

(c) Springer-Verlag GmbH Germany, part of Springer Nature 2019

\section{Correction to: Plant Reproduction https://doi.org/10.1007/s00497-019-00378-5}

Table 4 in the original publication reports incomplete genotype names in the column "Cross" and wrong codes in the column "Generation". The wrong names and codes have been corrected. The correct table 4 is reported below. The errors do not affect the interpretation and conclusions of the study.

The authors apologize for any inconvenience.

Publisher's Note Springer Nature remains neutral with regard to jurisdictional claims in published maps and institutional affiliations.
The original article can be found online at https://doi.org/10.1007/ s00497-019-00378-5.

Maurizio Sabatti

sabatti@unitus.it

Muriel Gaudet

murielvirginie.gaudet@cnr.it

Niels A. Müller

niels.mueller@thuenen.de

Birgit Kersten

birgit.kersten@thuenen.de

Cosimo Gaudiano

cos.gaudiano@gmail.com

Giuseppe Scarascia Mugnozza gscaras@unitus.it

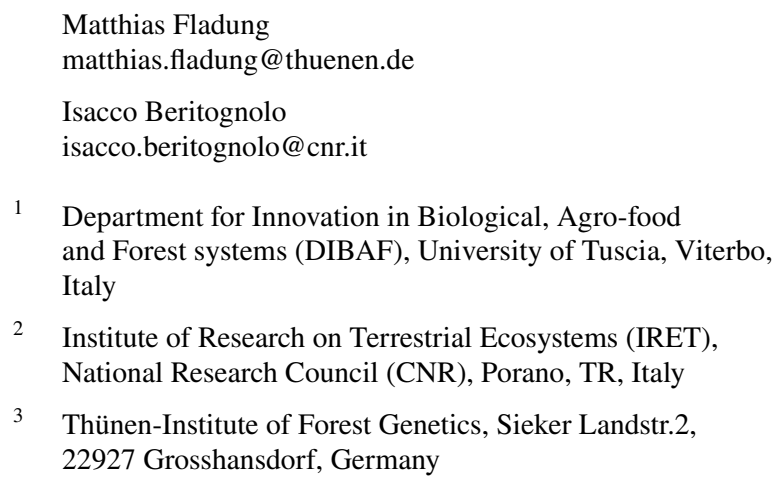

Isacco Beritognolo isacco.beritognolo@cnr.it

1 Department for Innovation in Biological, Agro-food and Forest systems (DIBAF), University of Tuscia, Viterbo, Italy

2 Institute of Research on Terrestrial Ecosystems (IRET), National Research Council (CNR), Porano, TR, Italy

3 Thünen-Institute of Forest Genetics, Sieker Landstr.2, 22927 Grosshansdorf, Germany 
Table 4 Germination experiments carried out on progenies from controlled crosses in a $P$. $\times$ canescens ('6K6' $\times$ 'VV') pedigree.

Germination capacity (GC) observed at 16 days (2012) or 20 days (2009 and 2010) after sowing. ANOVA and HSD post-hoc tests were performed within each year, after exclusion of samples with no replicates.

Different letters indicate significant difference between group means. St. Dev., standard deviation (in brackets)

\begin{tabular}{|c|c|c|c|c|c|}
\hline & Cross & Generation & Replicates & GC mean & (St. Dev.) \\
\hline \multirow[t]{7}{*}{ Year 2009} & ' $111 \mathrm{KV}$ ’ ‘40KV' & $\mathrm{F}_{2}$ & 2 & 43.42 & (11.32) \\
\hline & '111KV’×‘90KV' & $\mathrm{F}_{2}$ & 3 & 38.89 & $(3.18)$ \\
\hline & '90KV' & OP & 3 & 37.85 & (4.34) \\
\hline & $' 111 \mathrm{KV}$ ' $\times{ }^{\prime} \mathrm{VV}$ ' & $\mathrm{BC}_{P t}$ & 2 & 28.76 & $(18.25)$ \\
\hline & ‘6K6’ × ‘40KV’ & $\mathrm{BC}_{P a}$ & 1 & 20.00 & - \\
\hline & '90KV' & $\mathrm{S}_{1}$ & 1 & 20.00 & - \\
\hline & ANOVA $p 0.603^{\mathrm{NS}}$ & & & & \\
\hline \multirow[t]{6}{*}{ Year 2010} & '111KV’×‘VV' & $\mathrm{BC}_{P t}$ & 6 & $96.67^{\mathrm{a}}$ & $(10.44)$ \\
\hline & ' $111 \mathrm{KV}$ ’ ‘40KV' & $\mathrm{F}_{2}$ & 6 & $95.17^{\mathrm{a}}$ & $(1.72)$ \\
\hline & ‘6K6’ × ‘40KV' & $\mathrm{BC}_{P a}$ & 6 & $94.83^{\mathrm{a}}$ & $(2.14)$ \\
\hline & '91KV' & $\mathrm{S}_{1}$ & 6 & $64.83^{\mathrm{b}}$ & $(0.82)$ \\
\hline & '90KV' & $\mathrm{S}_{1}$ & 2 & $42.78^{\mathrm{c}}$ & $(24.36)$ \\
\hline & ANOVA $p 4.47 \mathrm{e}^{08} * * *$ & & & & \\
\hline \multirow[t]{11}{*}{ Year 2012} & '45S $1-90 \mathrm{KV} '$ & $\mathrm{OP}$ & 3 & $85.82^{\mathrm{a}}$ & $(5.22)$ \\
\hline & ' $48 \mathrm{~S}_{1-90 \mathrm{KV}}$ ' & $\mathrm{OP}$ & 3 & $73.25^{\mathrm{ab}}$ & $(3.56)$ \\
\hline & '90KV' & $\mathrm{OP}$ & 3 & $71.76^{\mathrm{ab}}$ & $(5.31)$ \\
\hline & '6K6’ × ‘40KV' & $\mathrm{BC}_{P a}$ & 3 & $71.25^{\mathrm{ab}}$ & $(1.91)$ \\
\hline & $' 111 \mathrm{KV}$ '× $40 \mathrm{KV}$ ' & $\mathrm{F}_{2}$ & 3 & $67.18^{\mathrm{abc}}$ & (11.19) \\
\hline & '111KV’×`VV' & $\mathrm{BC}_{P t}$ & 3 & $66.42^{\mathrm{abc}}$ & $(3.80)$ \\
\hline & ‘6K6’’ ${ }^{\prime} \mathrm{VV} ’$ & $\mathrm{~F}_{1}$ & 3 & $65.83^{\mathrm{abc}}$ & $(18.65)$ \\
\hline & ‘ $6 \mathrm{~K} 6 ’ \times ‘ 90 \mathrm{KV}$ ’ & $\mathrm{BC}_{P a}$ & 3 & $54.14^{\mathrm{bc}}$ & (12.38) \\
\hline & '90KV' & $\mathrm{S}_{1}$ & 3 & $49.44^{\mathrm{bc}}$ & $(9.68)$ \\
\hline & $' 111 \mathrm{KV}$ ' $\times$ '90KV' & $\mathrm{F}_{2}$ & 3 & $43.33^{\mathrm{c}}$ & $(8.01)$ \\
\hline & ANOVA $p 0.0014 * *$ & & & & \\
\hline
\end{tabular}

The level of significance of the $p$ value is indicated as: NS non-significant; $* * p \leq 0.01 ; * * * p \leq 0.001$ 